\title{
Auditing Physicians to Improve Their Infection Control Behavior?
}

\author{
C. Ruef
}

The role of hand disinfection before and after patient contact is undisputed. Numerous studies demonstrate poor compliance with indications for hand disinfection in the hospital. Some of these studies have also shown a difference in compliance between nurses and physicians, with physicians typically performing worse than nurses. As the transmission of nosocomial pathogens by contaminated or colonized hands of health care workers is the most important risk factor for nosocomial infections, the importance of hand disinfection cannot be overstated. During recent years, many countries have started campaigns that focus on hand disinfection. This topic is also central to the "Clean Care is Safer Care" campaign of the World Health Organization.

While some success has been achieved with hospitalwide or nation-wide or even world-wide campaigns, there is still room for improvement. Getting the physicians to perform better in this regard remains a major challenge.

In this issue of Infection, Howard et al. [1] report an interesting approach. They carried out an infection control audit using a "clean practice protocol." This protocol consists of the observation of infection control activities during the patient contact of physicians, the proper placement of patients with certain nosocomial pathogens, such as MRSA, instrument cleaning after use, and notes contamination. Using a scoring system for each of the observed activities, the authors calculated an overall score of infection control activities for each patient consultation.
The result of the first audit, which was performed undisclosed, was poor, demonstrating a hand disinfection rate of $28 \%$. The authors then went on to teach the surgeons and had great success with this, as illustrated by a hand disinfection rate of $87 \%$ during the repeat audit after teaching activities. All other parameters also showed significant improvement in comparison to the baseline audit.

I think that this study is quite interesting, providing some food for thought regarding the options that we have to improve the infection control performance of physicians in the hospital. There are some methodological questions regarding the validity of the second audit. I wonder whether the second audit could be performed as undisclosed as the first one. However, the idea is original and might warrant validation by other colleagues in other hospitals.

\section{Reference}

1. Howard DPJ, Williams C, Sen S, Shah A, Daurka J, Bird R, Loh A, Howard A: A simple effective clean practice protocol significantly improves hand decontamination and infection control measures in the acute surgical setting. Infection 2009; 37: 34-38.

Infection 2009; 37: 1

DOI 10.1007/s15010-009-3109-Y

Published online: January 23, 2009 